\title{
DIAGNÓSTICO DIFERENCIAL DE DOR TORÁCICA NA EMERGÊNCIA
}

\section{DIFFERENTIAL DIAGNOSIS OF THORACIC PAIN IN EMERGENCY}

\author{
Marcos Roscine Andrade Leite ${ }^{1}$ \\ Ingridy Michely Gadelha do Nascimento ${ }^{2}$ \\ Talina Carla da Silva ${ }^{3}$ \\ Macerlane de Lira Silva ${ }^{4}$ \\ Ankilma do Nascimento Andrade Feitosa ${ }^{5}$
}

RESUMO: Objetivo: Realizar uma revisão integrativa com destaque nos diagnósticos diferenciais de dor torácica na emergência. Metodologia: Trata-se de uma pesquisa bibliográfica do tipo exploratória, no qual foram utilizadas as bases de dados PUBMED e MEDLINE para obter os dados de artigos e periódicos nacionais e internacionais, referentes aos diagnósticos diferenciais de dor torácica no prontosocorro. Resultados e Discussão: O quadro da dor torácica corresponde a uma dor profunda, em aperto ou opressão, com uma duração de dez a vinte minutos, sendo

\footnotetext{
${ }^{1}$ Graduando em Medicina pela Faculdade Santa Maria - FSM, Cajazeiras, Paraíba.

${ }^{2}$ Graduanda em Enfermagem pela Faculdade Santa Maria - FSM, Cajazeiras, Paraíba.Membro do Grupo de estudos e pesquisa na área da saúde do idoso. Membro do Grupo de Pesquisalnovações tecnológicas no ensino superior: desafios contemporâneos.

${ }^{3}$ Doutora em Ciências da Saúde pela Escola de Enfermagem da Universidade de São Paulo (EEUSP)-2019. Mestre em Saúde Pública pela Universidade Estadual da paraíba (UEPB)2014.Bacharelado e Licenciatura em Enfermagem formada pela Universidade Estadual da Paraíba (UEPB) - 2011. Docente da Faculdade Santa Maria e Coordenadora da Pós-Graduação da Faculdade de Santa Maria de Cajazeiras. Especialista em Saúde Coletiva pela Faculdade Integrada de Patos. Integrante do Núcleo de Estudos e Pesquisas Epidemiológicas (NEPE/UEPB). Membro do Grupo de Pesquisa Avaliação dos Serviços de Saúde (CNPq). Membro do Grupo de Pesquisa Vulnerabilidade, Adesão e Necessidades em Saúde Coletiva? (USP).

${ }^{4}$ Possui graduação em Enfermagem pela FSM -Cajazeiras, especialização em política e gestão do cuidado com ênfase no apoio matricial pela UFPB e mestrado em Saúde Coletiva pela UNISANTOS. Docente da Faculdade Santa Maria de Cajazeiras, membro do Comitê de Ética em Pesquisa da Universidade Federal de Campina Grande, Campus cajazeiras e voluntário da Associação de Pais e Amigos dos Excepcionais de Cajazeiras.

${ }^{5}$ Doutora em Ciências da Saúde pela Faculdade de Medicina do ABC. Possui Mestrado (2010) e Licenciatura (2009) em Enfermagem Pela Universidade Federal da Paraíba. Especialização em Auditoria em Serviços de Saúde. Especialização em Saúde da Família pela UFPB. Especialista em Processos Educacionais na Saúde pelo Sírio libanês. Graduação em Enfermagem pela Faculdade Santa Emília de Rodat (2005). Docente da Faculdade Santa Maria-PB, dos cursos de Medicina e Enfermagem. Compõe o Banco de Avaliadores do Sistema Nacional de Avaliação da Educação Superior (BASis).
} 
localizada na região precordial e desencadeada por estresse ou exercício, no qual a dor em região mandibular deve ser valorizada. Portanto, o paciente com essa queixa na emergência necessita de um o diagnóstico rápido e preciso, tendo em vista primordialmente o manuseio e tratamento das condições que oferecem risco de morte ao mesmo. Durante a abordagem de um paciente com desconforto precordial no pronto-socorro, o emergencista deve dá preferência ao atendimento das pessoas com dores torácicas, além de fornecer metas diagnósticas e terapêuticas organizadas na sala de emergência, objetivando rapidez, alta qualidade de cuidados, eficiência e redução de custos. O objetivo inicial é destacar uma síndrome coronariana aguda (SCA), sem esquecer outras causas que podem colocar em risco o quadro clínico do paciente, tais como: embolia pulmonar, pneumotórax, pericardite etc. É de extrema importância que o pronto-socorro esteja equipado e organizado com materiais de reanimação cardiopulmonar e uma equipe ágil e competente de forma a priorizar o atendimento ao usuário. Quanto mais precoce for diagnosticado uma Síndrome Coronariana Aguda (SCA), melhor será o prognóstico e a qualidade de vida para os pacientes. Em acréscimo ao tratamento, foram criadas as Unidades de Dor Torácica (UDT), cujo objetivo principal consiste no reconhecimento de forma imediata de um Infarto Agudo do Miocárdio (IAM) e na realização de uma pronta terapia de reperfusão. Conclusão: Através deste estudo, foi possível concluir a dificuldade do emergencistaem estabelecer um diagnóstico exato, visto que a dor torácica representa uma gama de diagnósticos diferenciais. Mesmo com tanta experiência profissional, o médico na maioria das vezes acaba por menosprezar os sintomas do paciente, aumentando assim a mortalidade e os erros diagnósticos.

Palavras chave: Diagnóstico. Dor no Peito. Emergências. Exame Físico. Registros Médicos.

ABSTRACT: Objective: Toperformanintegrativereviewofdifferential diagnoses ofchestpain in emergency. Methodology: This isan exploratory bibliography icresearch, conductedfromtheconsultationoftheBireme site, usingthedatabases: Lilacs (Latin American and Caribbean Health Sciences Literature), from which there sultswereobtained. data from national and international article sandjournalsregardingdifferential diagnoses ofemergencychestpain. Results And Discussion: chestpainis a deep, tighteningoroppressivepainlasting 10 to 20 minutes, located in the precordialregiontriggeredby stress orexercise, andpain in the mandibularregionshouldbevalued. Therefore, the patient with this emergency complaintneeds a prompt and accurate diagnosis, primarilyaimingatthehandlingandtreatmentofconditionsthat pose a riskofdeathto the patient. In the approach of a patient in the emergency roomwithprecordial discomfort, the emergency roomshouldgive preference to the careof patient swithchestpain, provideorganized diagnosticandtherapeuticgoals in theemergencyroom, aimingatspeed, high qualityofcare, efficiencyandcostreduction. The initialobjectiveistohighlightanacutecoronarysyndrome (ACS), withoutforgettingother causes thatmayendangerthepatient'sclinicalcondition, such as pulmonaryembolism, pneumothorax, pericarditis, it isextremelyimportantthattheemergencyroomisequippedandorganized withcardiopulmo naryresuscitationmaterialsandanagileandcompetentteamtoprioritizethecareofpatients 
withchestpain. The earlieran Acute Coronary Syndrome (ACS) isdiagnosed, the better the prognosis and quality oflife for patients. Toaddto the treatment were created the Chest Pain Units (UDT), whosemain objective is the immediaterecognitionofan Acute Myocardiallnfarction (AMI) and promp treperfusiontherapy. Throughthisstudy, it waspossibletoconcludetheemergentist'sdifficulty in establishinganaccuratediagnosis, sincechestpainrepresents a range ofdifferential diagnoses. Evenwithsomuch professional experience, the doctor mostoftenoverlooks the patient'ssymptoms, thusincreasing mortality and mis diagnosis.

Keywords: Diagnosis. Chestpain. Emergencies. Physical Exam. Medical Records. 


\section{INTRODUÇÃO}

A dor torácica tem como descrição clássica, uma sensação de dor, irritação, formigamento ou sentimento de enclausuração, encontrada na retroesternal, que vai se irradiando para o ombro, braço esquerdo, braço direito, pescoço ou mandíbula, frequentemente acompanhada de vômitos, náuseas ou dispnéia. O paciente também pode relatar um quadro clínico atípico como mal-estar, fraqueza ou apenas sudorese. Essa dor geralmente dura em torno de dez a vinte minutos (TEXEIRA, 2013).

Atualmente um dos maiores desafios para o profissional médico é um paciente com dor torácica no pronto atendimento, este sintoma é bastante frequente que pode advir de múltiplas causas e representar uma grande diversidade de diagnóstico e tratamento, que por muitas vezes leva o médico a ter dúvidas em diferenciar qual o tipo de dor presente. Portanto, o paciente com essa queixa na emergência precisa de um $\mathrm{o}$ diagnóstico rápido e preciso, objetivando primordialmente o manuseio e cuidados das condições que oferecem risco de óbito ao paciente (MARTINS, 2013).

Tanto as sociedades como a comunidade médica recomendam que os médicos emergencistas trabalhem defendendo a saúde e a segurança do paciente. Sendo assim, pacientes incapazes de apresentar um diagnóstico exato, utilizando da anamnese, do exame físico e do eletrocardiograma, os médicos mostram-se desacreditados com o dilema de aceitar ao hospital a grande parte dos pacientes com algum tipo de dor torácica que buscam a sala de emergência, ou de dar alta àqueles com menores possibilidades de desenvolver doença cardiovascular de risco. Dessa forma, grande parte dos médicos acabam internando grande parcela de todos os pacientes que tenham possibilidades de apresentar síndrome coronariana aguda (BASSAN, 2002).

As pesquisas mostram que cerca de seis milhões de pacientes buscam serviços de emergência todos os anos, o que correspondem a $5,5 \%$ de todos os 
atendimentos nesta área. De seis milhões de pacientes com algum tipo de dor torácica consultados nas unidades de emergência, quatro milhões são aceitos por suspeita de Síndrome Coronariana Aguda (SCA). Porém, devido às limitações da história clínica, do exame físico e do eletrocardiograma, vários pacientes são aceitos em leitos sem o diagnóstico exato (PESARO et al., 2007).

Uma característica das unidades de emergência é a presença de pacientes com o quadro clínico de risco iminente de morte, com pacientes estáveis e ambulatoriais, isto dificulta o diagnóstico de pacientes potencialmente graves. $\mathrm{O}$ manejo inicial desses pacientes deve ser feito sempre com a intenção de confirmar ou afastar tais hipóteses de diagnóstico. Dentre os casos mais comuns de dor torácica pontuamos: angina, infarto agudo do miocárdio (IAM), pericardite, dissecção de aorta, embolismo pulmonar e pneumonia (TEIXEIRA, 2013).

Uma história clínica e um exame físico criterioso para os pacientes que procuram o pronto socorro com queixa de dor torácica, tem um fator a mais de importância com relação aos exames complementares. Na maioria das vezes a análise de pacientes com algum tipo de dor torácica é feita de forma subjetiva e imprópria, mesmo sendo realizada por médicos com experiência profissional, mas que não seguem os protocolos de diagnósticos específicos. A equipe de enfermeiros e médicos devem estar atentos e treinados, usando de ferramentas adequadas para o manuseio de pacientes acometidos com esses sinais clínicos (FERREIRA et al., 2011).

Apesar dos avanços dos métodos de exames complementares para confirmação diagnóstica, a dor torácica continua sendo um grande desafio para toda a equipe médica. Quando um paciente chega na emergência queixando-se de dor torácica, a abordagem deve ser criteriosa, pois isso possui influência significativa para a redução da mortalidade e dos custos hospitalares relacionados a estas situações. Na verdade, nenhum sistema público de saúde consegue arcar com os gastos dos exames complementares, restringindo assim sua solicitação. (MARTINS, 2013).

Em torno da dificuldade do médico em encontrar o diagnóstico preciso, devido a dor torácica representar um sintoma referente a várias doenças, que vão desde o infarto agudo do miocárdio até uma dissecção de aorta (que deve ser sempre 
lembrada no paciente com dor torácica na emergência), a equipe médica deve ter o diagnóstico correto do tipo de dor torácica que aquele paciente venha a possuir e realizar as intervenções necessárias para estabilizar o quadro clinico do paciente, reduzindo os custos hospitalares, fornecendo estratégia diagnóstica e terapêutica organizada, objetivando rapidez, e dispondo de leitos exclusivos para atender os mesmos, equipados com monitor cardíaco, (RCP) equipamento para parada cardiorrespiratória, eletrocardiograma e laboratório para dosar as enzimas cardíacas.

Desse modo, o objetivo desse trabalho é realizar uma revisão integrativa da literatura com destaque nos diagnósticos diferenciais de dor torácica no prontosocorro, apresentando as estratégias diagnósticas e terapêuticas feitas nas salas de emergência, que buscam rapidez, qualidade de cuidados, eficiência e redução de gastos, destacando a Síndrome Coronariana Aguda (SCA) e ressaltando a prioridade de atendimento aos pacientes com dores torácicas.

\section{METODOLOGIA}

Para a execução do presente estudo foi adotada a revisão integrativa do tipo exploratória, no qual a pesquisa bibliográfica foi realizada nas bases de dados PUBMED e MEDLINE, utilizando os descritores: diagnóstico, exame físico, dor torácica, história clínica e emergência, nos quais mostraram grandes resultados para alcançar os objetivos propostos. A pesquisa teve como base a seguinte pergunta norteadora: "Quais os possíveis diagnósticos diferenciais diante da dor torácica na emergência apresentado pela literatura?".

A revisão integrativa identifica, analisa e sintetiza resultados de estudos independentes sobre uma temática específica, incluindo estudos experimentais e não experimentais, possibilitando emergir vários aspectos de um mesmo fenômeno. Sua aplicabilidade se dá não somente pela elaboração de protocolos, procedimentos, políticas, como também âmbito do pensamento crítico que a prática diária necessita (SOUZA et al., 2010; GOMES et al., 2014). 
$\mathrm{Na}$ realização da pesquisa científica, foram adotados como critérios de inclusão: estudos feitos em humanos (filtro "specieshumans"), publicados entre os anos de 2002 a 2014, sendo obtidos os dados de artigos e periódicos nacionais e internacionais referentes aos diagnósticos diferenciais de dor torácica na emergência. Após leitura completa e seleção dos artigos adequados aos critérios de inclusão predefinidos, foi feita uma organização de seus conteúdos de acordo com os quesitos: ano, tipo de publicação e métodos/técnicas utilizadas, objetivos, essência do conteúdo, produção do conhecimento/resultados e as principais recomendações feitas pelos autores dos estudos, excluindo os artigos que não atenderam aos critérios de inclusão e/ou aos objetivos do trabalho já mencionados, após a leitura completa dos mesmos.

Posteriormente a qualificação do projeto, a análise dos dados e os passos subsequentesforam iniciados. Esta etapa deve ser efetuada com criticidade, buscando explicações para os diferentes resultados, que podem ser até contraditórios entre os estudos (MENDES et al., 2008). Para tanto, neste trabalho foi feita uma interpretação crítica do conteúdo, com listagem dos principais fatores socioeconômicos associados à referidos na literatura. Ademais, levou-se em consideração o nível de evidência dos estudos.

\section{RESULTADOS E DISCUSSÃO}

\subsection{AVALIAÇÃO CLÍNICA INICIAL}

No momento da análise ou verificação dos pacientes com dor torácica aguda, o médico deve priorizar uma série de procedimentos relacionados ao prognóstico e a conduta imediata. Mesmo antes de tentar se aproximar de um quadro clínico compatível com determinada doença, devem ser analisadas questões de alta prioridade que incluem: Estabilidade Clínica; o paciente está precisando de uma intervenção imediata de colapso circulatório ou insuficiência respiratória? 
Prognóstico imediato; se o paciente está com um quadro clínico estável, qual a probabilidade de risco para que possa ter uma patologia ameaçadora à vida, como SCA, embolismo pulmonar ou dissecção aórtica? Segurança das opções de triagem; se os riscos de patologias ameaçadoras à vida forem pequenos, seria correto dar alta ao paciente para tratamento ambulatorial ou o paciente deve ficar em análise e fazer testes adicionais para seguimento de seu respectivo tratamento? (TEXEIRA, 2013).

As diretrizes do National Heart Attack Alert Program afirmam que os pacientes com os sintomas típicos que chamam bastante atenção, devem ser avaliados de forma imediata pelos enfermeiros de triagem e encaminhados para avaliação mais precisa, os sintomas são: dor torácica, em aperto ou opressão, com duração de dez a vinte minutos, dor que se irradia para o pescoço, mandíbula, ombros, dorso ou ambos os braços; indigestão, ou azia, náuseas, e/ou vômito associados a desconforto torácico; dispnéia persistente, fraqueza, tontura e perda de consciência (TEXEIRA, 2013).

\subsection{ABORDAGEM CLÍNICA}

$\mathrm{Na}$ abordagem de um paciente no pronto-socorro com desconforto precordial, o emergencista deve dá preferência ao acolhimento de pacientes com muita dor torácica, disponibilizar metas diagnóstica e terapêutica organizada na sala de emergência, objetivando praticidade, alta qualidade de cuidados, eficiência e redução de gastos. O objetivo inicial é destacar uma síndrome coronariana aguda (SCA), sem se esquecer de outras causas que possam colocar em risco o quadro clínico do paciente, tais como: embolia pulmonar, pneumotórax, pericardite etc. (MARTINS, 2013).

Caso o paciente não esteja necessitando de uma abordagem imediata em razão de colapso circulatório ou insuficiência respiratória, a avaliação médica deve começar com uma história clínica bem criteriosa e que detalhe as características gerais da dor, quando foi que se iniciou, certificar se essa dor tem irradiação, qual a 
duração dos sintomas, questionar se algum parente da família teve infarto, e solicitar um exame que enfatize os sinais vitais e a condições cardiovasculares. Esse questionamento deve estar centrado no rastreamento das patologias mais comuns que ameaçam a vida: o infarto agudo do miocárdio (IAM), o embolismo pulmonar e a dissecção aórtica aguda. Dentre esses três diagnósticos, o mais comum em salas de emergência é a SCA (Síndrome Coronariana Aguda). Como a SCA representa aproximadamente um quinto das causas de dor torácica nas salas de pronto atendimento e tem uma significativa morbimortalidade, a medida inicial desses pacientes é sempre feita no sentido de confirmar ou afastar esse diagnóstico (TEXEIRA, 2013). Não esquecer: uma dor torácica com característica mais intensa, súbita e com duração aproximada de dez a vinte minutos, junto com outros sinais e sintomas como: dispneia, sudorese, palidez cutânea e náuseas, frequentemente representa um infarto agudo do miocárdio (MARTINS, 2013).

\subsection{CAUSAS COMUNS DE DOR TORÁCICA}

O estudo realizado por Texeira (2013) aponta que aproximadamente $20 \%$ dos pacientes com dor torácica aguda que dá entrada no setor de emergência possui IAM ou angina instável (AI), uma pequena parcela dos demais apresenta outros problemas ameaçadores a vida como dissecção de aortaeembolismo pulmonar, porém a maioria destes recebem alta sem um diagnóstico definido ou com um diagnóstico de patologia não cardíaca, que inclui refluxo esofágico, pneumonia, dentre outros.

- As maiores causas de dor torácica estão resumidas no quadro 1. 
Quadro 1 - Principais Causas de Dor Torácica.

\begin{tabular}{|c|c|c|c|}
\hline Sistema & Síndrome & Descrição Clínica & Características \\
\hline \multirow{4}{*}{ Cardíaco } & Angina & $\begin{array}{lr}\text { Pressão } & \text { torácica } \\
\text { retroesternal, queimação } & \text { quadia } \\
\text { ou peso; } & \text { irradia } \\
\text { ocasionalmente } & \text { para } \\
\text { pescoço, mandíbula, } \\
\text { epigástrio, ombros ou } \\
\text { braço esquerdo. }\end{array}$ & $\begin{array}{l}\text { Precipita-se pelo exercício, } \\
\text { tempo frio ou estresse } \\
\text { emocional; duração }<2-10 \\
\text { minutos. }\end{array}$ \\
\hline & $\begin{array}{l}\text { Angina } \\
\text { Instável }\end{array}$ & $\begin{array}{l}\text { A mesma da angina, } \\
\text { porém pode ser mais } \\
\text { intensa. }\end{array}$ & $\begin{array}{l}\text { Dura geralmente < } 20 \\
\text { minutos. Menor tolerância } \\
\text { para o esforço. }\end{array}$ \\
\hline & IAM & $\begin{array}{l}\text { A mesma da angina, } \\
\text { porém pode ser mais } \\
\text { intensa. }\end{array}$ & $\begin{array}{l}\text { Início súbito, geralmente } \\
\text { durando } 30 \text { minutos ou } \\
\text { mais. Frequentemente } \\
\text { associado à dispnéia, } \\
\text { fraqueza, náuseas e } \\
\text { vômitos. }\end{array}$ \\
\hline & Pericardite & $\begin{array}{lr}\text { Dor aguda, } & \text { pleurítica, } \\
\text { agravada } & \text { pelas } \\
\text { modificações } & \text { na posição; } \\
\text { duração } & \text { altamente } \\
\text { variável. } & \end{array}$ & Atrito pericárdico \\
\hline \multirow[t]{2}{*}{ Vascular } & $\begin{array}{l}\text { Dissecção } \\
\text { aórtica }\end{array}$ & $\begin{array}{l}\text { Dor excruciante, lacerante, } \\
\text { de início abrupto, na parte } \\
\text { anterior do tórax, } \\
\text { frequentemente se } \\
\text { irradiando para o dorso. }\end{array}$ & $\begin{array}{l}\text { Dor marcadamente intensa } \\
\text { e refratária; geralmente } \\
\text { ocorre em um contexto de } \\
\text { hipertensão ou de um } \\
\text { distúrbio subjacente do } \\
\text { tecido conjuntivo, como a } \\
\text { síndrome de Marfan. }\end{array}$ \\
\hline & $\begin{array}{l}\text { Embolismo } \\
\text { pulmonar }\end{array}$ & $\begin{array}{l}\text { Início súbito de dispnéia e } \\
\text { dor, geralmente pleurítica, } \\
\text { com infarto pulmonar. }\end{array}$ & $\begin{array}{lr}\text { Dispnéia, } & \text { taquipnéia, } \\
\text { taquicardia e } & \text { sinais de } \\
\text { insuficiência } & \text { cárdica } \\
\text { direita. } & \end{array}$ \\
\hline Pulmonar & $\begin{array}{l}\text { Pleurite } \\
\text { e/ou } \\
\text { Pneumoni } \\
\text { a }\end{array}$ & $\begin{array}{l}\text { Dor pleurítica, geralmente } \\
\text { breve, sobre a área } \\
\text { envolvida. }\end{array}$ & $\begin{array}{l}\text { Dor pleurítica associada à } \\
\text { dispnéia. }\end{array}$ \\
\hline $\begin{array}{l}\text { Gastrointe } \\
\text { stinal }\end{array}$ & $\begin{array}{l}\text { Refluxo } \\
\text { esofágico }\end{array}$ & $\begin{array}{l}\text { Desconforto queimação } \\
\text { subesternal epigástrico. }\end{array}$ & $\begin{array}{l}\text { Agravada por refeições } \\
\text { pesadas e por se deitar } \\
\text { após alimentar-se. }\end{array}$ \\
\hline
\end{tabular}

Fonte: TEXEIRA, 2013, p.8. 


\subsection{MÉTODOS E DIAGNÓSTICO}

É de extrema importância que o pronto-socorro esteja equipado e organizado com materiais de reanimação cardiopulmonar e disponha de uma equipe ágil e competente, de forma a dar prioridade ao atendimento de todos os pacientes com dor torácica, sendo o resultado inicial excluir uma síndrome coronariana aguda (SCA), não se esquecendo das causas potencialmente catastróficas. O tratamento precoce dos pacientes com infarto agudo do miocárdio é uma medida salvadora de vidas e deve ser rapidamente iniciado, quando diagnosticado, em até 30 minutos a partir do momento de chegada do paciente ao hospital, sabendo que quanto mais precoce for o diagnóstico de uma SCA, muito maior será a probabilidade de salvar o miocárdio e melhorar o prognóstico dos pacientes (BASSAN, 2002).

\subsubsection{Eletrocardiograma}

O exame de eletrocardiograma é fundamental e deve ser realizado imediatamente em até dez minutos após a chegada do paciente na urgência. Não vai haver mudanças na maioria dos pacientes que se mostram com dor torácica, desses pacientes que apresentem Eletrocardiograma (ECG) normal, 5\% terão síndrome coronariana aguda, não podendo ser o único exame complementar utilizado para dar certeza ou confirmar o afastamento do diagnóstico dessa doença. ECG seriados, com alguns intervalos de três a quatro horas nas primeiras doze horas de internação, aumentando ainda mais (para até 95\%) o diagnóstico de IAM. A sensibilidade desse exame na internação para IAM é de 45\% - 60\% (supra de ST). Sendo assim, $50 \%$ dos pacientes não são diagnosticados com infarto agudo do miocárdio apenas com um exame simples de ECG feito no atendimento; sendo importante elaborar toda uma fiscalização adequada e realizar os ECG seriados, que 
devem ser adquiridos no intervalo máximo de três horas após o primeiro (MARTINS, 2013).

\subsubsection{Avalição Inicial do ECG}

O ECG mostra um aumento do segmento ST maior que $1 \mathrm{~mm}$ em duas ou mais derivações contíguas: provavelmente o diagnóstico é de SCA com elevação do segmento ST. Quando ECG não mostra elevação do segmento ST maior que $1 \mathrm{~mm}$ em duas derivações contíguas, mas tenha sintomas compatíveis, está diante de uma SCA sem elevação do segmento ST, neste caso é preciso solicitar as enzimas seriadas para saber se é um caso de angina instável (Al), se as enzimas cardíacas não estiverem elevadas; ou se é um IAM sem elevação do segmento ST, caso as enzimas cardíacas estejam elevadas. O ECG sem nenhuma anormalidade não descarta uma doença coronariana, ele é essencial, traz informações importantes e de grande valor, especialmente os ECGs seriados (MARTINS, 2013).

- A seguir, pode-se observar no quadro 2 a representação de um eletrocardiograma com supra de ST. 
Quadro 2 - Representação de um Eletrocardiograma com Supra de ST.

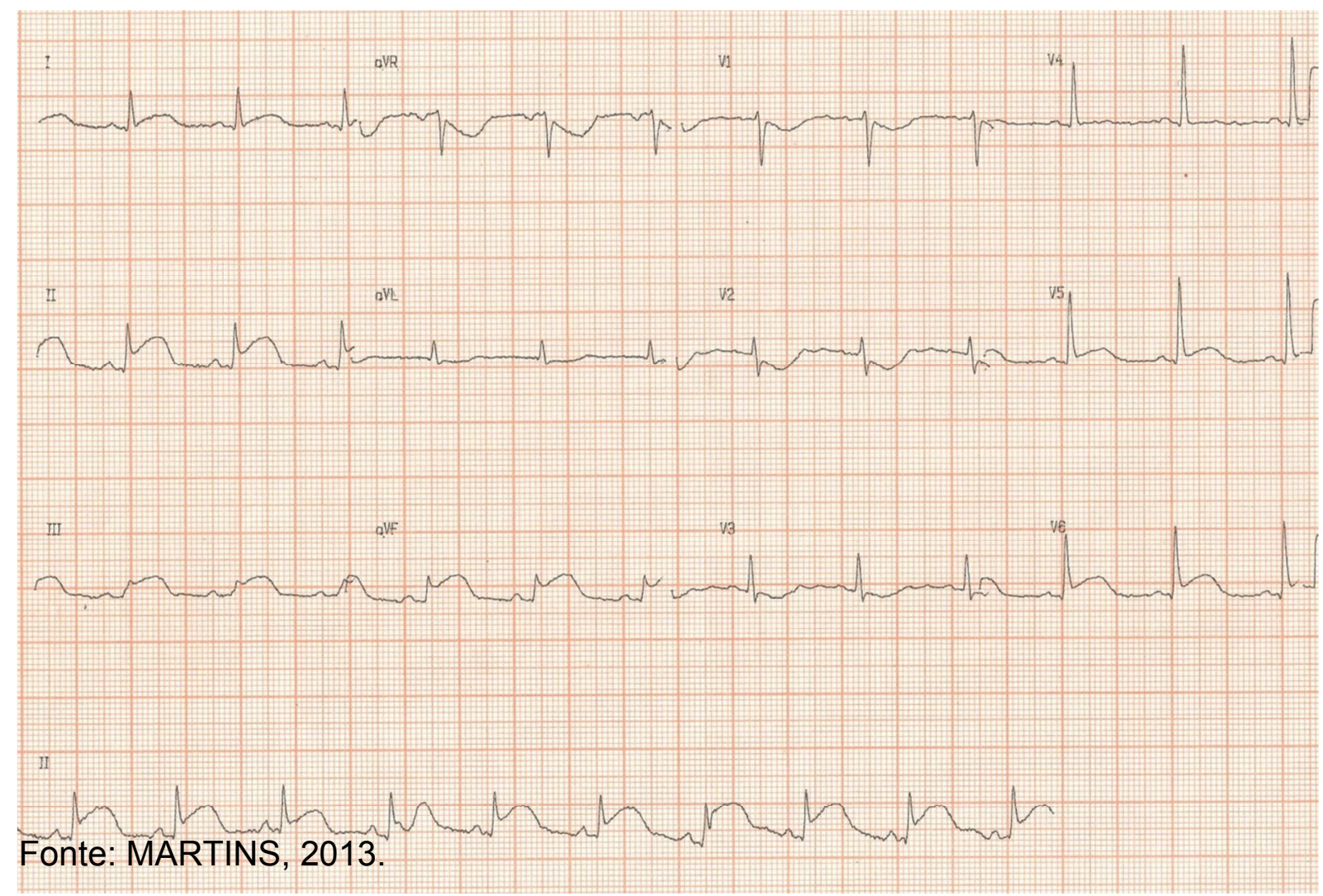

\subsubsection{Marcadores de Necrose do Miocárdio}

Segundo o estudo realizado por Texeira (2013) as troponinas cardíacas T e I são os marcadores laboratoriais mais sensíveis e específicos de lesão miocárdica. Essas troponinas estão na maioria das vezes ausentes no sangue de uma pessoa normal. Em média a troponina $\mathrm{T}$ tem sensibilidade de 96,9\%, especificidade de $94,5 \%$, valor preditivo negativo de $99,8 \%$ para o diagnóstico de IAM. Por isso é o melhor marcador para o diagnóstico de infarto do miocárdio, superando a CKMB (Creatinoquinase). A melhor opção é dosar as troponinas no internamento e repetir após seis horas a doze do início da dor. Esse exame feito de forma isolada não é suficiente para diagnosticar um infarto. Os marcadores de lesão miocárdica devem ser medidos em todos os pacientes com suspeita clínica de síndrome coronariana 
aguda. Mesmo não tendo o resultado de imediato das enzimas cardíacas, o paciente com quadro clínico sugestivo, dor típica, e alteração no ECG, deve ser tratado como SCA.

\subsubsection{Radiografia de Tórax e Tomografia de Tórax}

A radiografia de tórax possui uma maior eficácia na realização de um diagnóstico de dor torácica com origem não isquêmica como: pneumonia, pneumotórax, câncer de pulmão, derrame pleural etc. A tomografia computadorizada de tórax tem mais sensibilidade e especificidade do que a radiografia de tórax para diagnostico de doenças pleurais, do parênquima e vasculatura pulmonar. É mais utilizado para o diagnóstico de embolia pulmonare dissecção de aorta (BASSAN, 2002).

\subsubsection{Ecocardiograma}

A função do ecocardiograma (ECO) de repouso na avaliação do paciente com dor torácica, ainda não está sendo critério para confirmação do diagnóstico de SCA. Sua sensibilidade para diagnóstico de IAM varia de $70 \%$ a $95 \%$, mas tem falsos positivos bastantes elevados, o que torna baixo o valor preditivo positivo. Já o valor preditivo negativo varia de $85 \%$ - 95\%. Em pacientes com um ECO normal não agrega informação diagnóstica significativa, além daquelas fornecidas pela história e pelo ECG. Portanto, um ECO negativo não deve descartar um IAM ou angina de alto risco. O ecocardiograma tem fundamental importância no diagnóstico de várias doenças e é útil nos casos de derrame pericárdio, estenose aórtica e valvopatias, cardiomiopatia hipertrófica, dissecção de aorta (TEXEIRA, 2013). 


\subsection{TRATAMENTO}

Mesmo apresentando um exame de ECG e as enzimas cardíacas em níveis normais na internação, não se deve afastar a hipótese de uma doença cardíaca em um paciente com sintomas típicos de dor torácica, o que leva o responsável médico a solicitar internação hospitalar, (método tradicional). Isso traz enormes prejuízos ao sistema público de saúde e ao setor privado. Para tentar minimizar esse problema, estudos foram feitos na tentativa de traçar metas estratégicas seguras para o paciente, tendo uma ótima relação de custo-efetividade. Sendo então criadas as Unidades de Dor Torácica (UDT), um planejamento útil e custo-efetivo (MARTINS, 2013).

O objetivo básico das UDT é divulgar principalmente o reconhecimento de forma imediata do IAM e conceder ao paciente uma pronta terapia de reperfusão. Ao lado disso, o foco é afastar condições clínicas com potencial elevado de risco e de morte, como angina instável, dissecção de aorta, embolia pulmonar, pneumotórax hipertensivo, ruptura do esôfago, úlcera perfurada e pancreatite aguda. Uma vez na UDT, visto que as síndromes coronarianas agudas são definidas pela clínica, pela sintomatologia, sendo a principal sintomatologia a dor, dor constritiva, em aperto. Caso tenha dúvida se tratar de uma síndrome coronariana, deve-se manter o paciente em ambiente hospitalar, e realizar MOV - Monitorização, Oxigênio e Veia; MONAB - Morfina, Oxigênio, Nitrato, AAS e Betabloqueador (MARTINS, 2013).

Reconhecido um paciente com Síndrome Coronariana Aguda (SCA), não é preciso enzimas para começar a tratá-lo O eletrocardiograma deve sair em até 10 minutos, feito os exames, já se pode notar a diferença se é uma SCA com ou sem supra de ST, para ela ser considerado um supra, é preciso que haja o aumento de ao menos $2 \mathrm{~mm}$ em duas ou mais especificações. Na SCA com supra a artéria estará obstruída, e é feito a angioplastia, que é o tratamento de eleição e trombolíticos ou fibrinolíticos que são usados quando não há a possibilidade de uma angioplastia. O trombolítico mais comum é a estreptoquinase. Esse grupo de medicamento tem um melhor rendimento considerando que quanto mais nova for a 
síndrome (em até 3 horas), conforme o tempo vai passando, menor será sua eficácia. Deve-se atentar para as contraindicações dos trombolíticos, que são divididas em absolutas: AVC hemorrágico (em qualquer época da vida), TCE recente (até poucos meses), tumores intracranianos, Acidente Isquêmico Transitório (AIT - nos últimos 3 meses), suspeita de dissecção de aorta; e as relativas: menstruação, gravidez, uso de vafarina, crise hipertensiva (MARTINS, 2013).

Isso se tratando da síndrome com supra, mas quando se trata das síndromes sem supra, a artéria não está obstruída, então o tratamento é desenvolvido com AAS, clopidogrel, heparina, betabloqueador, internamento e cateterismo. $O$ cateterismo na SCA com supra de ST é um procedimento de urgência, e nas síndromes sem supra não é de urgência, porém mesmo assim não é recomendável liberar o paciente para casa, este deve ser internado, mantido em observação, e monitorado, para receber todo o aparato terapêutico e fazer o cateterismo mais ou menos programado, se baseando no tipo de SCA: baixo, médio ou alto risco. O de baixo risco fica internadoe medicado, pode ir até para o ambulatório depois de passada algumas horas em observação, se ele estiver bem, estável, poderá ir para o ambulatório e programar o cateterismo. O de médio risco se torna de internamento, sem muita pressa. Enquanto o de alto risco, já deve ser feito o cateterismo nas primeiras 24 a 48 horas, sendo chamado de cateterismo precoce.

\section{CONSIDERAÇÕES FINAIS}

Acerca do que foi pesquisado, compreende-se e torna-se claro a dificuldade do emergencista de estabelecer um diagnóstico exato, visto que a dor torácica representa uma gama de diagnósticos diferenciais. Mesmo com tanta experiência profissional, o médico na maioria das vezes acaba por menosprezar os sintomas do paciente, aumentando assim a mortalidade e erros diagnósticos. É importante ressaltar que, o objetivo primordial ao examinar um paciente com dor torácica é afastar causas potencialmente ameaçadoras a vida. Para isso é importante possuir um serviço de emergência estruturado, e uma equipe organizada, treinada, fazendo 
assim diagnósticos corretos e condutas adequadas, diminuindo os custos hospitalares.

\section{REFERÊNCIAS BIBLIOGRÁFICAS}

BASSAN, Roberto. Unidades de Dor Torácica. Uma Forma Moderna de Manejo de Pacientes com Dor Torácica na Sala de Emergência. Arquivos Brasileiros de Cardiologia. Rio de Janeiro, v.79, n² 2, p.196-202, 2002.

FERREIRA, A. M. C.; MADEIRA. M. Z. A. Revista Interdisciplinar Novafapi, Teresina, v.4, n.1, p.50-56, Jan-Fev-Mar. 2011.

GOMES, Isabelle Sena; DE OLIVEIRA CAMINHA, Iraquitan. Guia para estudos de revisão sistemática: uma opção metodológica para as Ciências do Movimento Humano. Movimento, v. 20, n. 1, p. 395-411, 2014.

MARTINS, H. S. Dor Torácica. In: MARTINS, H. S.; DAMASCENO, M. C. T.; AWADA, S. B. Pronto Socorro Medicina e Emergência. $3^{\circ}$ ed. São Paulo: Manole, 2013, cap. 50, p.421-431.

MENDES, Karina DalSasso; SILVEIRA, Renata Cristina de Campos Pereira; GALVÃO, Cristina Maria. Revisão integrativa: método de pesquisa para a incorporação de evidências na saúde e na enfermagem. Texto \& contexto enfermagem, v. 17, n. 4, p. 758-764, 2008.

PESARO, A. E. P. et. al. Síndromes coronarianas agudas: como fazer um diagnóstico correto na sala de emergência. Einstein. São Paulo, v. 5, n. 1, p. 80-4, junho, 2007.

SOUZA, Marcela Tavares de; SILVA, MICHELLY Dias da; CARVALHO, Rachel de. Revisão integrativa: o que é e como fazer. Einstein (São Paulo), v. 8, n. 1, p. 102-106, 2010.

TEIXEIRA, J. C. G. Dor Torácica-Abordagem na Unidade de Emergência. Unidade de Emergência Condutas em Medicina de Urgência. $3^{\circ}$ ed. São Paulo: Atheneu, 2013, cap.2, p.7-19. 